\title{
Combination and QCD Analysis of Charm Production Measurements in Deep-Inelastic ep Scattering at HERA
}

\author{
Katerina LIPKA* on behalf of the $\mathrm{H} 1$ and ZEUS Collaborations \\ Deutsches Elektronen-Synchrotron \\ Notkestrasse 85, D-22607, Hamburg, Germany \\ E-mail: katerina.lipka@desy.de
}

\begin{abstract}
Measurements of open charm production cross sections in deep-inelastic ep scattering at HERA from the $\mathrm{H} 1$ and ZEUS Collaborations are combined. Reduced cross sections for charm production are obtained in the kinematic range of photon virtuality $2.5 \leq Q^{2} \leq 2000 \mathrm{GeV}^{2}$ and Bjorken scaling variable $3 \cdot 10^{-5} \leq x \leq 5 \cdot 10^{-2}$. The combination method accounts for the correlations of the systematic uncertainties among the different data sets. The combined charm data together with the combined inclusive deep-inelastic scattering cross sections from HERA are used as input for a detailed NLO QCD analysis to study the influence of different heavy flavour schemes on the parton distribution functions. The optimal values of the charm mass as a parameter in these different schemes are obtained. The implications on the NLO predictions for $W^{ \pm}$and $Z$ production cross sections at the LHC are investigated. Using the fixed flavour number scheme, the running mass of the charm quark is determined.
\end{abstract}

The European Physical Society Conference on High Energy Physics

18-24 July, 2013

Stockholm, Sweden

${ }^{*}$ Speaker. 
Measurements of open charm production in deep-inelastic electron-proton scattering (DIS) at HERA provide important input for stringent tests of quantum chromodynamics (QCD). At HERA, charm quarks are predominantly produced by the boson-gluon-fusion process, which is sensitive to the gluon distribution in the proton. The mass of the charm quark, $m_{c}$, provides a sufficiently high scale necessary to apply perturbative QCD (pQCD). However, additional scales are involved in charm production, e.g. the virtuality, $Q^{2}$, of the exchanged photon in case of DIS and the transverse momenta, $p_{T}$, of the outgoing quarks. The presence of several hard scales complicates the QCD calculations for charm production. Depending on the details of the treatment of $m_{c}, Q$ and $p_{T}$, different approaches in $\mathrm{PQCD}$ have been formulated. Here, the massive fixed-flavour-number scheme (FFNS) and different implementations of the variable-flavour-number-scheme (VFNS) are considered.

At HERA, different techniques have been used to measure open charm production cross sections in DIS. The full reconstruction of charmed mesons, the long lifetime of heavy flavoured hadrons or their semi-leptonic decays are exploited. Measurements of charm production crosssections by the $\mathrm{H} 1$ and ZEUS experiments using different charm tagging techniques are combined. The details of the included measurements, combination procedure and the employed theory can be found in [1]. For the combination, the published cross sections in the restricted phase space regions of the individual measurements are extrapolated to the full phase space of charm production in a coherent manner by the use of FFNS calculations in next-to-leading order (NLO) QCD [2, 3]. The results are presented in terms of reduced cross sections, $\sigma_{\text {red }}^{c \bar{c}}$.

In the combination procedure, applied to 155 data points from 9 measurements, the correlated systematic uncertainties and the normalisation of the different measurements are accounted for such that one data set is obtained. There are in total 48 sources of correlated systematic uncertainties. The data show good consistency, with a $\chi^{2}$-value per number of degrees of freedom, ndof, of $\chi^{2} / \mathrm{ndof}=62 / 103$. The combined measurement has 52 points and covers the kinematic range of $2.5 \leq Q^{2} \leq 2000 \mathrm{GeV}^{2}$ and $3 \cdot 10^{-5} \leq x \leq 5 \cdot 10^{-2}$, with $x$ being the partonic fraction of the proton energy. Since different experimental techniques of charm tagging have been employed using different detectors and methods of kinematic reconstruction, this combination leads to a significant reduction of statistical and systematic uncertainties. At medium $Q^{2}$, a precision of $5 \%$ is achieved.

The combined data are compared to various QCD predictions produced by different theory groups. This comparison, described in detail in [1], tests the interplay between the gluon and/or charm distributions as obtained in different schemes and the charm treatment within each scheme, as well as the related choice of the central value for the respective charm-quark mass. In the zeromass variable-flavour-number-scheme (ZM-VFNS) [4] the charm quark mass is set to zero in the computation of the matrix elements and kinematics, and a threshold is introduced at $Q^{2} \approx m_{c}^{2}$, below which the charm production cross section is assumed to vanish. In the FFNS [5, 2], the charm quark is treated as massive at all scales, and is not considered as a parton in the proton. The number of active flavours is fixed to three, and charm quarks are assumed to be produced only in the hard scattering process. In the general-mass variable-flavour-number-schemes (GM-VFNS) [6] charm production is treated in the FFNS in the low $Q^{2}$ region, where the mass effects are largest, and in the ZM-VFNS approach at high $Q^{2}$, where the effect of resummation is most noticeable. At intermediate scales an interpolation is made between the FFNS and the ZM-VFNS. The freedom introduced by choosing an interpolation approach prevents a clear interpretation of the charm mass 

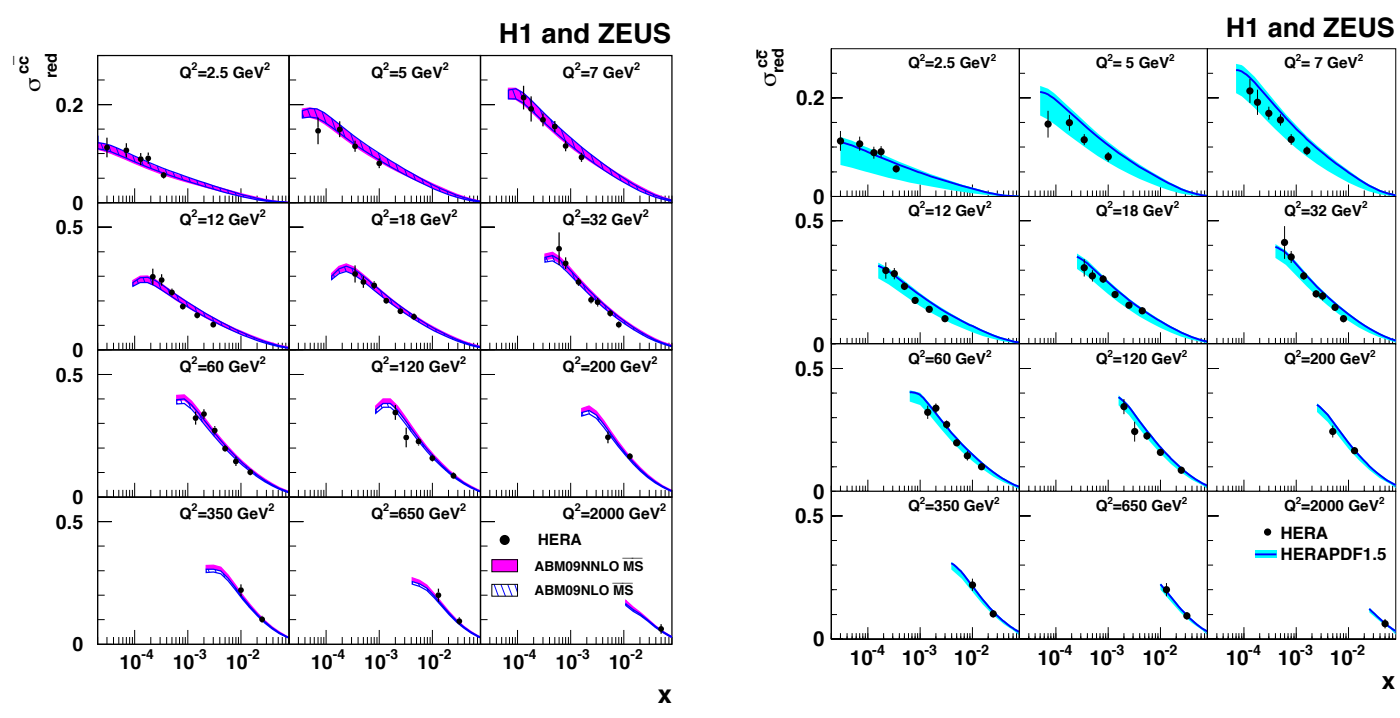

Figure 1: Combined $\sigma_{\text {red }}^{c \bar{c}}($ filled circles) with its total uncertainty (error bars) as a function of $x$ for fixed values of $Q^{2}$. The data are compared to the FFNS predictions (left) at NLO (hashed band) and NNLO (filled band) using running charm-quark mass in the $\overline{M S}$ scheme. The data are compared to NLO predictions in VFNS (right) based on HERAPDF1.5, using $M_{c}=1.4 \mathrm{GeV}$. The uncertainty band shows the full PDF uncertainty which is dominated by the variation of $M_{c}$.

in terms of a specific renormalisation scheme. Therefore the charm mass appearing in the GMVFNS is treated as a mass parameter, $M_{c}$, of the individual model. In Fig. 1 the data are compared to the prediction [7] in FFNS at NLO and NNLO, based on the running-mass scheme for both the coefficient functions and the parton density functions (PDFs). This scheme has the advantage of reducing the sensitivity of the cross sections to higher order corrections, and improving the theoretical precision of the mass definition. The uncertainties on the prediction include the uncertainties on the running charm quark mass which dominate at small $Q^{2}$. The predictions at NLO and NNLO are very similar and describe the data well in the whole kinematic range of the measurement. In Fig. 1, the combined data are also presented in comparison to QCD prediction in GM-VFNS at NLO using HERAPDF1.5 parton distributions, indicating the sensitivity of the charm data to the choice of the charm quark mass for the PDF determination.

Sensitivity of the combined $\sigma_{\text {red }}^{c \bar{c}}$ to the charm-quark mass used in the PDFs is studied by including the combined charm data into a QCD analysis together with the combined inclusive DIS cross sections [9], using different models of charm production in DIS. The analysis is performed with the HERAFitter [10] program, with details of the PDF parametrization described in [1]. The resulting gluon, valence and sea distributions are shown in Fig. 2 compared to PDFs obtained using the inclusive DIS data only. By including charm data, the uncertainties on the gluon and the charm distributions are reduced, mostly due to improved constraints on the charm mass. Correspondingly, the uncertainty on the $u$ - and $d$-quark distribution functions is reduced. The sensitivity of the combined $\sigma_{\text {red }}^{c \bar{c}}$ data to the charm quark mass in the PDFs is demonstrated in Fig. 3, where the $\chi^{2}$ of the PDF fit to inclusive DIS data and to those including charm measurements is presented as a 

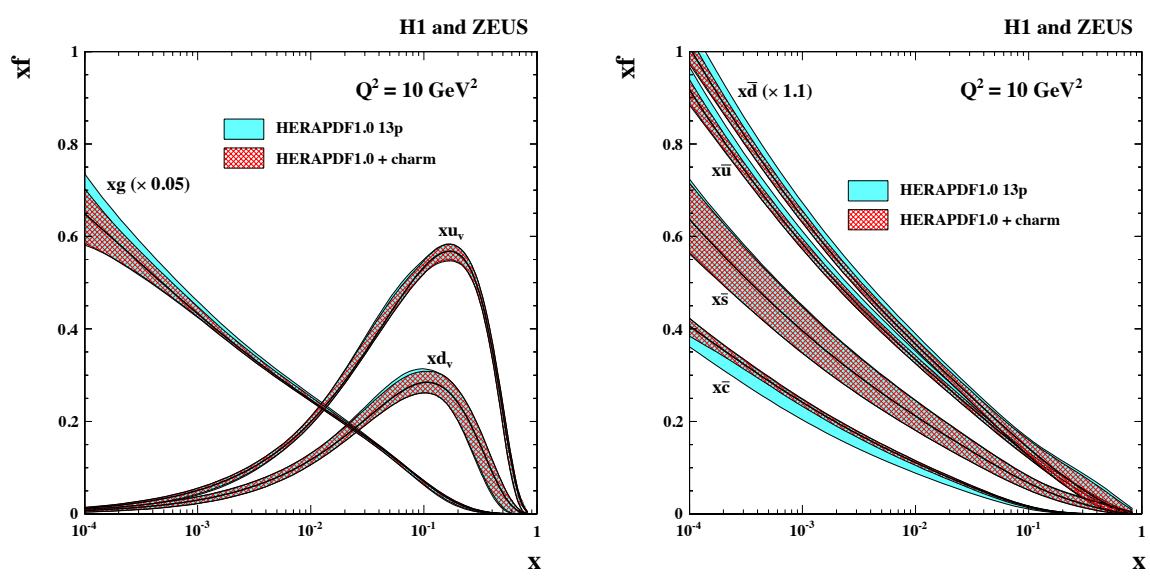

Figure 2: Parton density functions for valence quarks and the gluon (left) and for sea anti-quarks (right) obtained from the combined QCD analysis of the inclusive DIS data and $\sigma_{\text {red }}^{c \bar{c}}$ (dark shaded bands) as a function of $x$ at $Q^{2}=10 \mathrm{GeV}^{2}$. For comparison the results of the QCD analysis of the inclusive DIS data only are also shown (light shaded bands). The gluon distribution function is scaled by a factor 0.05 and the $d$-quark distribution function is scaled by a factor 1.1 for better visibility.
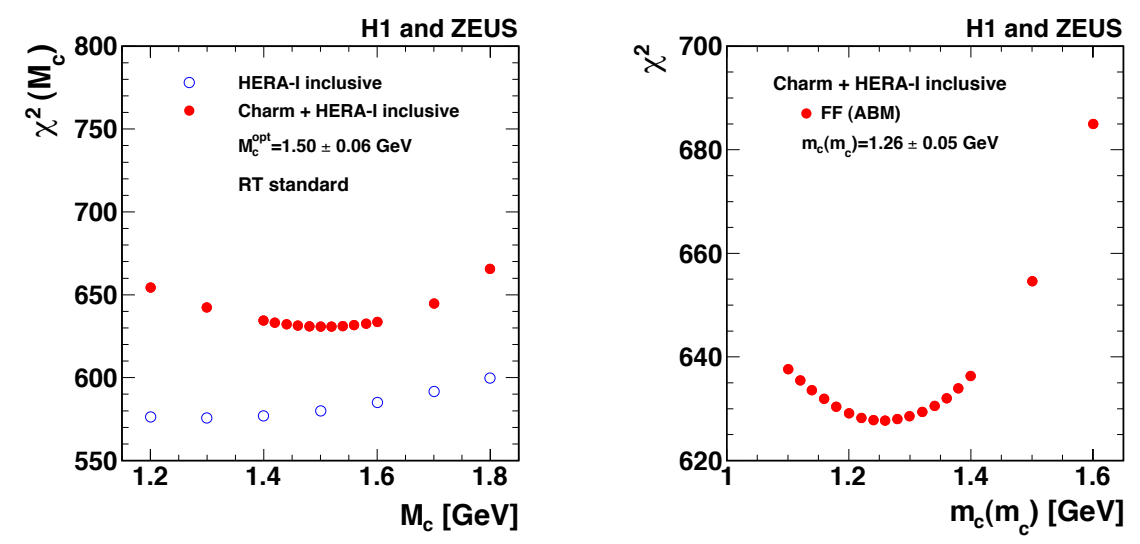

Figure 3: Left: the values of $\chi^{2}$ for the PDF fit to the combined HERA DIS data in VFNS as a function of $M_{c}$. The open symbols indicate the results of the fit to inclusive DIS data only. The results of the fit including the combined charm data are shown by filled symbols. Right: the values of $\chi^{2}$ for the PDF fit to the combined HERA DIS data including charm measurements as a function of the running charm quark mass $m_{c}\left(m_{c}\right)$. The FFNS scheme is used, where the charm quark mass is defined in the $\overline{M S}$ scheme.

function of $M_{c}$. Once the charm data are included in the fit, the minimum of $\chi^{2}$ is more pronounced as in the case of inclusive DIS data only. A similar $\chi^{2}$ scan is performed in the QCD analysis of the inclusive and charm data, using FFNS coefficient functions for charm production in the $\overline{M S}$ mass scheme. The result is shown in Fig. 3 and represents the determination of the running mass of the charm quark in the $\overline{M S}$ scheme at NLO. With experimental and PDF uncertainties, originating from the variation of model and parametrization assumptions as well as the uncertainty on the value 
of $\alpha_{S}$, the value of $m_{c}\left(m_{c}\right)=1.26 \pm 0.05_{\text {exp }} \pm 0.03_{\text {mod }} \pm 0.02_{\text {param }} \pm 0.02_{\alpha_{S}} \mathrm{GeV}$ is obtained. This result is consistent with an earlier result at NLO [8] and the world average [11] of $m_{c}\left(m_{c}\right)$ at NNLO.

Using different variants of the GM-VFNS, the optimal values of charm mass in each scheme, $M_{c}^{o p t}$, is obtained, as illustrated in Fig. 4. The values of $M_{c}^{o p t}$ differ for the different schemes. However, once an $M_{c}^{o p t}$ is used to evaluate the PDF in the corresponding VFNS scheme, the predictions of benchmark processes at the LHC using these different PDFs are very similar. In Fig. 4 the NLO prediction [12] for $W^{+}$boson production in proton-proton collisions at the LHC with a center-ofmass energy of $7 \mathrm{TeV}$ is presented as a function of $M_{c}$ in the PDFs using various heavy flavour schemes. When choosing the corresponding $M_{c}^{\text {opt }}$ for the schemes considered, the uncertainty due to the charm treatment in PDFs is reduced from $7 \%$ to below $2 \%$.
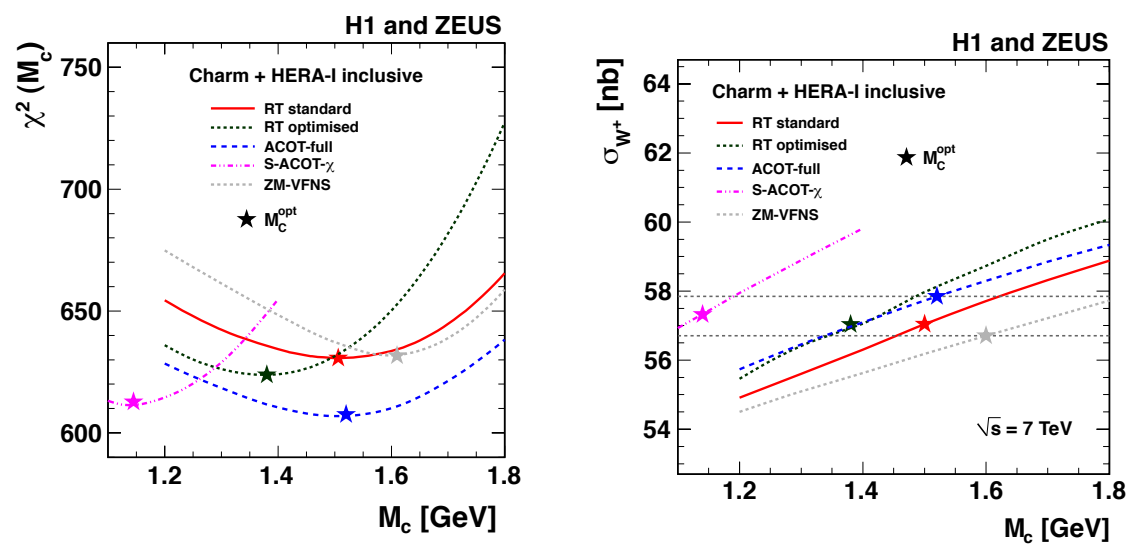

Figure 4: The values of $\chi^{2}\left(M_{c}\right)$ (left) for the PDF fit to the combined HERA inclusive DIS and charm measurements and corresponding NLO predictions (right) for $W^{+}$production cross sections at the LHC for $\sqrt{s}$ $=7 \mathrm{TeV}$ as a function of $M_{c}$ used in the corresponding PDF fit. The different lines represent predictions for different implementations of the VFNS. The values of $M_{c}^{o p t}$ for each scheme and corresponding predictions are indicated by the stars. The horizontal dashed lines show the resulting spread of the predictions when choosing $M_{c}=M_{c}^{o p t}$.

In addition to charm production cross sections, $\sigma_{\text {red }}^{c \bar{c}}\left(Q^{2}, x\right)$, the shapes of the kinematic distributions of charmed mesons could provide important information on the value of charm quark mass and also on the fragmentation model parameters. For the first time, the $D^{* \pm}$ cross section measurements as a function of the lepton and the meson kinematics were combined [13], using the measurements by the H1 [14] and ZEUS [15] collaborations. In Fig. 5 the results are shown as a function of the transverse momentum, $p_{T}\left(D^{*}\right)$ and pseudorapidity, $\eta\left(D^{*}\right)$, as compared to the NLO calculation [3]. The precision of the current calculation is mostly limited by estimation of the missing higher-order contribution, mimicked by an independent variation of the renormalization and factorization scales by a factor of two up and down, and is much lower than the experimental accuracy.

\section{References}

[1] [H1 and ZEUS Collaborations], Eur. Phys. J. C 73 (2013) 2311 [arXiv:1211.1182] . 

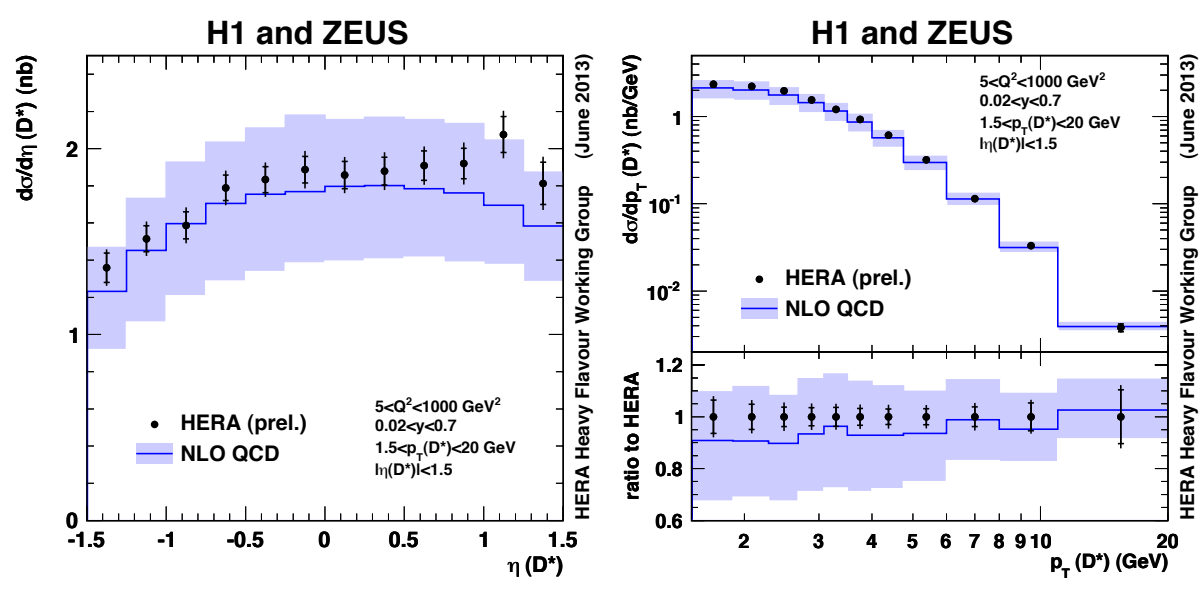

Figure 5: Combined $D^{* \pm}$ production cross section (filled symbols) as a function of the meson pseudorapidity, $\eta\left(D^{*}\right)$ (left), and transverse momentum, $p_{T}\left(D^{*}\right)$ (right). The inner error bars indicate the uncorrelated part of the uncertainties. The outer error bars represent the total uncertainties. The theory predictions and their uncertainty is shown by the shaded band. The ratio of theory to data $p_{T}\left(D^{*}\right)$ distribution is shown in the right bottom plot.

[2] S. Riemersma, J. Smith and W. L. van Neerven, Phys. Lett. B347, (1995) 143 [hep-ph/9411431].

[3] B. W. Harris and J. Smith, Phys. Rev. D57, (1998) 2806 [hep-ph/9706334].

[4] G. C. Collins and W.-K. Tung, Nucl. Phys. B278, (1986) 934.

[5] E. Laenen et al., Phys. Lett. B291, (1992) 325; E. Laenen et al., Nucl. Phys. B392, (1993) 162; E. Laenen et al., Nucl. Phys. B392, (1993) 229.

[6] M. A. G. Aivazis, F. I. Olness and W.-K. Tung, Phys. Rev. D50, (1994) 3085 [hep-ph/9312318]; M. Buza, et al., Nucl. Phys. B472, (1996) 611 [hep-ph/9601302];

J. C. Collins, Phys. Rev. D58, (1998) 094002 [hep-ph/9806259];

R. S. Thorne, Phys. Rev. D73, (2006) 054019 [hep-ph/0601245];

S. Forte et al., Nucl. Phys. B834, (2010) 116 [arXiv:1001.2312];

R. D. Ball et al. [NNPDF Collaboration], Nucl. Phys. B849, (2011) 296 [arXiv:1101.1300];

R. S. Thorne, arXiv:1201.6180.

[7] S. Alekhin and S. Moch, Phys. Lett. B699, (2011) 345 [arXiv:1011.5790].

[8] S. Alekhin et al, Phys. Lett. B 718 (2012) 550 [arXiv:1209.0436].

[9] [H1 and ZEUS collaborations], JHEP 1001:109 (2010) [arXiv:0911.0884].

[10] HERAFitter-0.2.1, http ://projects.hepforge.org/herafitter.

[11] K. Nakamura et al. [Particle Data Group], J. Phys. G37, (2010) 075021.

[12] J. Campbell and R. K. Ellis, MCFM-5.8, Phys. Rev. D60, (1999) 113006 [he-ph/9905386];

T. Carli et al., "APPLGRID-1.2.4", Eur. Phys. J. C66, (2010) 503 [arXiv:0911.2985].

[13] [H1 and ZEUS collaborations], H1-prelim-13-141, ZEUS-prel-13-002.

[14] [H1 Collaboration], Phys. Lett. B686, (2010) 91; [H1 Collaboration], Eur. Phys. J. C71 (2011) 1769.

[15] [ZEUS Collaboration], DESY-13-054. 